\title{
Design and Implementation of Nanotechnology QCA Geometric Greedy Router
}

\author{
Lamjed Touil (iD, Ismail Gassoumi (iD), and Abdellatif Mtibaa (iD) \\ Laboratory of Electronic and Microelectronic Faculty of Sciences at Monastir, University of Monastir, Monastir, Tunisia \\ Correspondence should be addressed to Ismail Gassoumi; gassoumiismail@gmail.com
}

Received 19 May 2021; Revised 15 August 2021; Accepted 2 September 2021; Published 15 September 2021

Academic Editor: Jit S. Mandeep

Copyright (C) 2021 Lamjed Touil et al. This is an open access article distributed under the Creative Commons Attribution License, which permits unrestricted use, distribution, and reproduction in any medium, provided the original work is properly cited.

\begin{abstract}
This paper presents an optimized geometric greedy router (GGR) based on quantum dot cellular automata (QCA) technology. The proposed structure of GGR is based on a spanning tree of the network. This type of communication does not require an IP address. It uses only local information and can be used in many communication devices. In this paper, we first describe the principal components of the router and then we present their QCA architecture. The QCA technology is the most likely alternative to replace conventional circuits (CMOS) due to their very low power consumption and high processing speed. To consider integration with other complex circuit, we have utilized QCA clock-phase-based technique for the proposed design architecture. The results obtained using the QCA designer tool exhibit the superiority of the presented architecture over the existing designs. The proposed structure shows a reduction of $30 \%$ reduction in occupied space. The power dissipation rate of the proposed design is analyzed by QCAPro tool to approve its reliability.
\end{abstract}

\section{Introduction}

The complementary metal oxide semiconductor (CMOS) technology has many defects, such as short channel effect and high power consumption; it cannot, therefore, continue to follow Morrie's law by increasing the number of devices per chip [1]. It creates the need for nanoscale devices, with high-performance rates and low power consumption. These recent nanoscale devices have the potential to enable progressions in different domains such as industrial, biomedical, and military fields. For these types of applications, classical communication networks, such as IP routing, are not suitable. In fact, these types of applications are very greedy for size, energy, and memory consumption [2]. The design of a geometric greedy router using the quantum dot cellular automata (QCA) can present an alternative to resolve these problems.

The quantum dot cellular automata (QCA) technology is one of the important emerging nanotechnologies that have attracted much researcher's attention in recent years. Such advantages led researchers to develop several projects detailing the construction of QCA circuits. In the recent years, several circuits such as address [3, 4], sequential circuits $[2,5]$, switching circuits $[6,7]$, reversible logic circuit $[8,9]$, and memories $[10,11]$ have been designed. On the contrary, to cope with the challenge of IOT, big data domains and the need to achieve data rates of up to multiple gigabits per second require the use of more efficient routing algorithms to avoid network bottlenecks. In the greedy routing method, the main aim is to achieve another routing process by having each participant store only a partial knowledge based on the network.

In the recent years, different studies of geometric greedy router have been proposed [12].

This routing method requires very efficient electronic support. In this paper, we present an optimized architecture of geometric greedy router based on QCA technology.

The paper is organized as follows. Section 2 presents the basic concept of QCA. Section 3 explains briefly the technique of the tree-based greedy routing. Section 4 covers the proposed architectures of the QCA geometric greedy router. Section 4 presents the simulation results of the proposed work along with discussion. Power dissipation analysis is carried out in Section 5. We conclude the paper with Section 6. 


\section{Preface to Quantum Dot Cellular Automata}

The basic QCA cell is illustrated in Figure 1. The QCA cell can be considered as a square including four quantum dots which are occupied by a pair of electrons. The cell comprises four quantum dots and two mobile electrons which are able to confine electric charges. These electrons tend to locate themselves and arranged diagonally in the cell. The repulsive force between two electrons pushes them as far as possible. There are two polarization states, which present logic " 0 " and "1," as shown in Figure 1 [13].

In QCA technology, the polarization of adjacent cells is performed by coulomb interaction, as shown in Figure 2(b). Polarization $(P)$ is defined as the following expression:

$$
\text { Polarization }(p)=\frac{(P 1+P 3)-(P 2+P 4)}{(P 1-P 2+P 3+P 4)} \text {. }
$$

The polarization of the electrons in the quantum dots are presented as $P 1, P 2, P 3$, and $P 4$ (Figure 2(a)). Equation (1) presents the value of polarization of QCA cell. If an electron is present, $P i=1$, otherwise it is equal 0 .

Inverter and majority gates are the essential logic element in the QCA circuits which are realized by some QCA cells, as shown in Figure 3 [13]. The majority gate can be used to generate an "AND" or an "OR" logic, by putting one of the inputs as " 0 " and " 1 ," respectively.

The QCA clocking system synchronization is used for controlling the potential barriers between the adjacent quantum dots. The QCA clocking system consists of four phases, as illustrated in Figure 4. The phases are switch, hold, release, and relax [14]. The barriers are raised during the switch phase, and a cell is influenced by the polarization of its adjacent cells. The barriers are high in the hold phase, and the polarization of the cell is retained. During the release phase, the barriers are lowered, and the cell loses the polarity. During the relaxing phase, the cell is nonpolarized.

In QCA, there are two different forms of crossover: coplanar and multilayer crossovers (Figure 5(a) and 5(b)). While multilayer crossover requires at least three layers for implementation, coplanar crossover uses only one layer. Multilayer QCA circuits consume much less area as compared to coplanar circuits.

\section{Greedy Router}

In geometric routing, a rather simple stateless distributed algorithm routes messages from node to node along a source-destination path, based only on position information (node coordinates). Only local information needs to be stored in each network node. A greedy routing algorithm forwards every packet to the node in the one-hop neighborhood, which lies closest to the final destination. In GR, virtual coordinates are assigned to each of the nodes of a network. The coordinate distribution is based on other ad hoc network protocols or sensors, such as greedy perimeter stateless routing (GPSR) [15]. In [16], the authors have proposed the use of virtual coordinates in NoGeo routing, the creation of the virtual coordinate assignment protocol (VCAP), and the use of conformal maps to find the coordinates [17]. In GR, a greedy technique is used. As the next hop, the neighbor node with the shortest distance to the destination node is selected.

The problem with geometric greedy routing occurs when a packet is in the case that the distance between the current node and the destination is smaller than the distance between its neighbors and the destination. The solution for this problem can be based on the face routing method [18] or using a greedy embedding method [19]. The embedding method can be done using multiple dimensions in Euclidean space $[20,21]$ or using multiple trees $[22,23]$ to improve routing performance. In our work, we used the method proposed in [24]. This method starts with the creation of a spanning tree and the adoption of a set of coordinates to each node of the network. Figure 6 presents this step. It consist of

(i) The creation of spanning tree (Figure 6(a))

(ii) Assignation of a number (from 1 to $n$ ) for the children of each node (Figure 6(b))

(iii) Give numbers to the children of each node (from 1 to $\mathrm{n}$ )

(iv) Assign $(0, \ldots, 0)$ to the root node

(v) A number previously is given to each child, replacing the first zero of their coordinates (Figure 6(c))

The tree depth presents the number of coordinates will be attributed to the nodes. The number of bits allocated to represent the coordinates in the router implementation is proportional to the number of children of the node. During the process, several steps must be established. When the router structure is created, the embedding tree has already been established and each generated node is aware of the coordinate set (CS) of its neighbors; the node forward the received packet to the neighbor which has the smallest possible distance to the destination. The processes are repeated until the packet reaches its final destination. Algorithm 1 presents the following steps.

The distance between nodes is computed according to the number of hops that must travel in the tree through the nodes, taking into consideration the common ancestors of neighbor nodes and the number of nonzero coordinates in each set.

\section{Architecture of the QCA Geometric Greedy Router}

Figure 7 shows the block diagram of the geometric greedy router.

Two tasks must be accomplished in the distance calculator module. The first task is based on the distance calculator algorithm (Algorithm 1). The model of the first part of the distance calculator is depicted in Figure 8. The second task is reserved for the destination node and the distance comparator determination.

The architecture of the greedy router is composed of several logic gates such as OR, XOR, NAND, and multiplexer. The optimized QCA layouts of these logic modules are presented in Figure 9. 


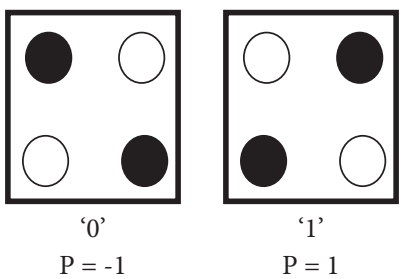

FIgURE 1: Schematics of the basic QCA cells.
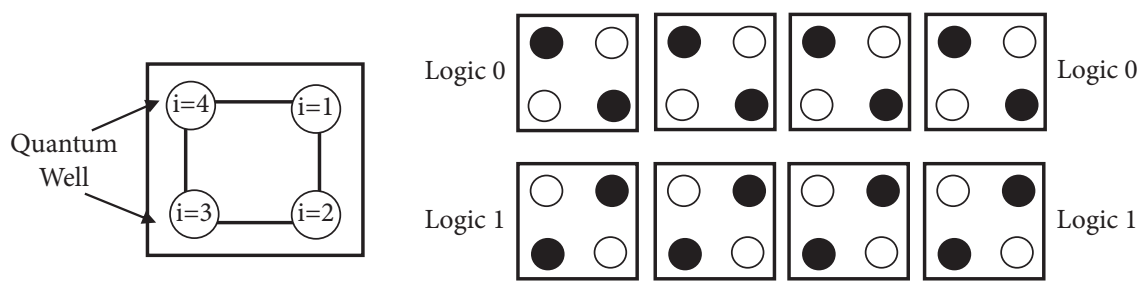

Figure 2: Propagation of binary information.
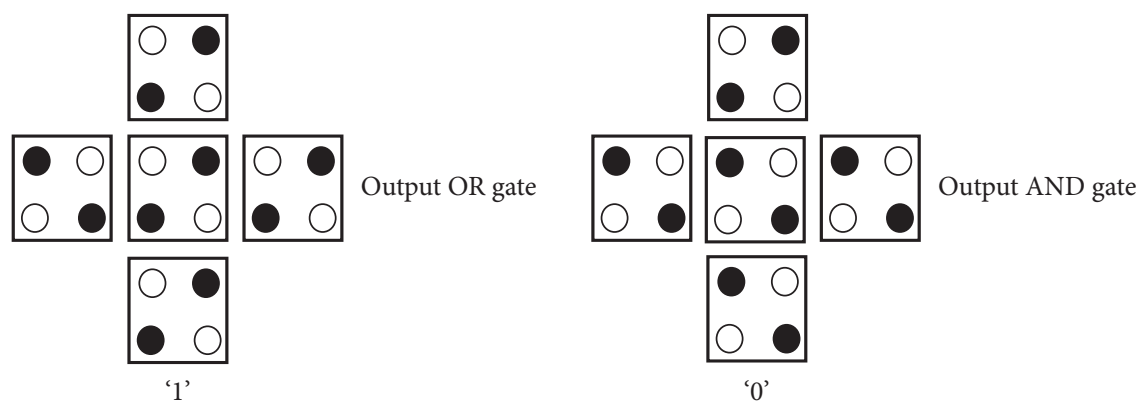

FIgUre 3: Realization of logical OR and AND gates.

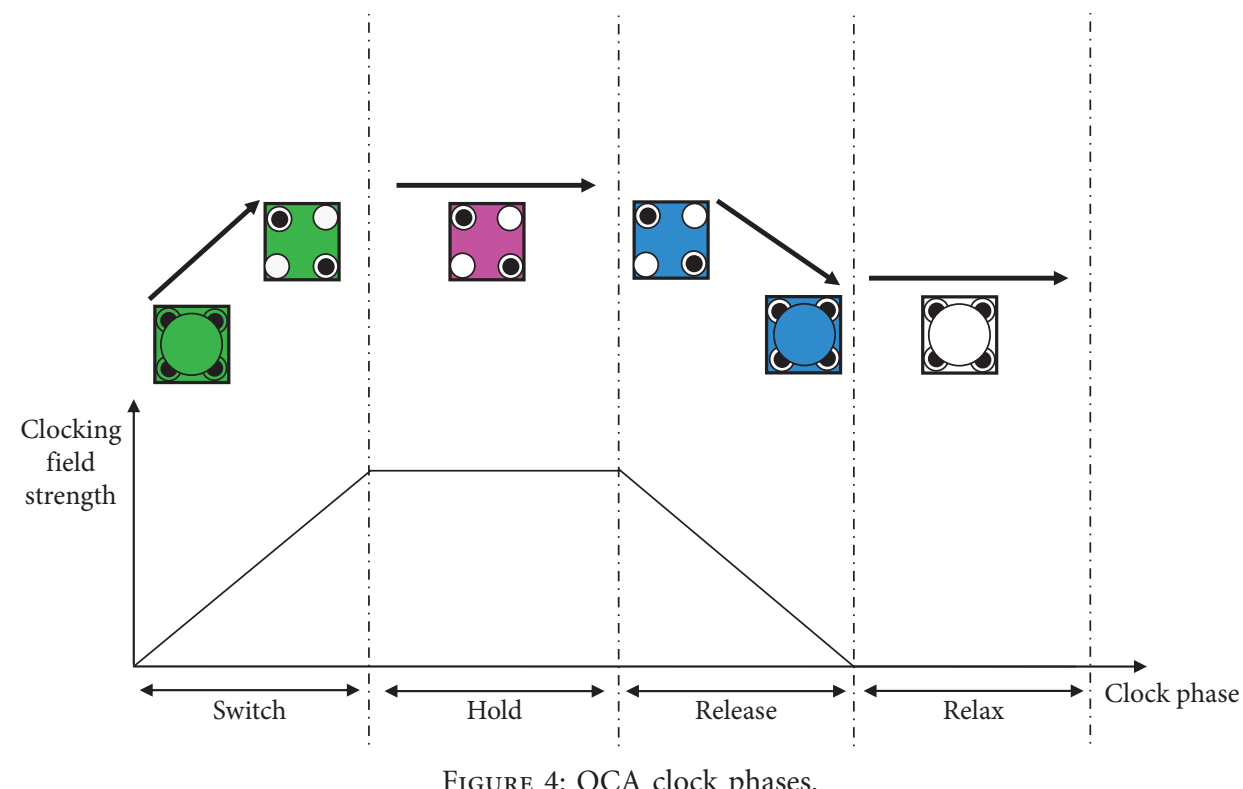

FIgURE 4: QCA clock phases. 


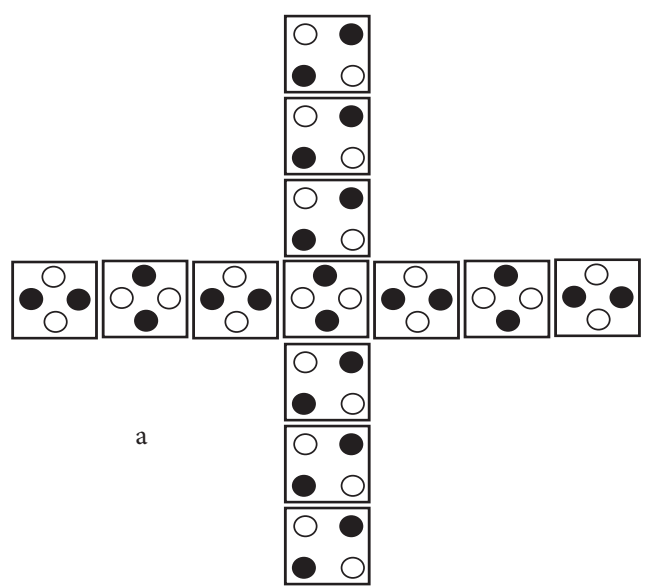

(a)

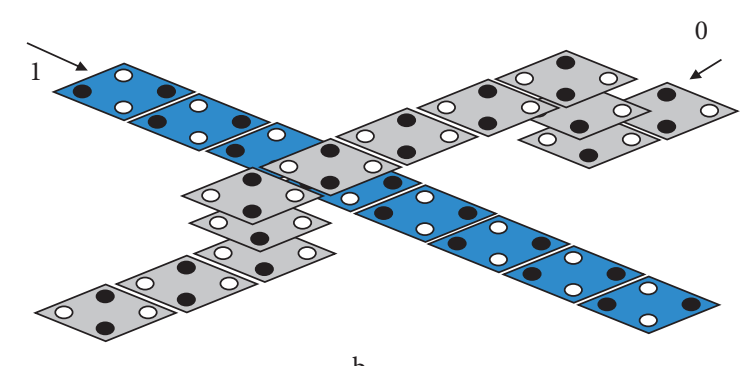

b

(b)

FIgURE 5: Signal crossover schemes: (a) coplanar crossing; (b) multilayer crossing.

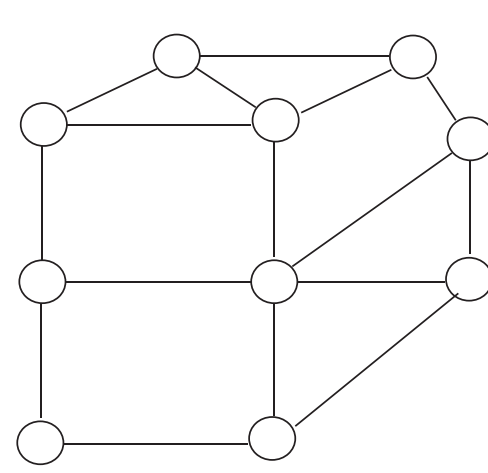

(a)

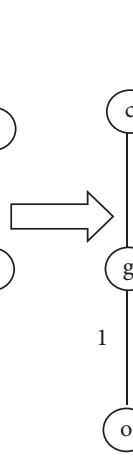

(g)--

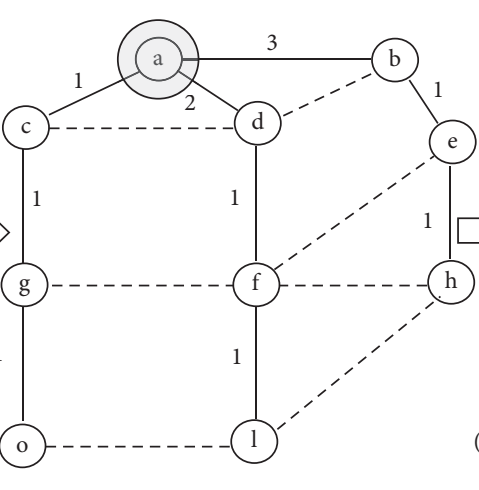

(b)

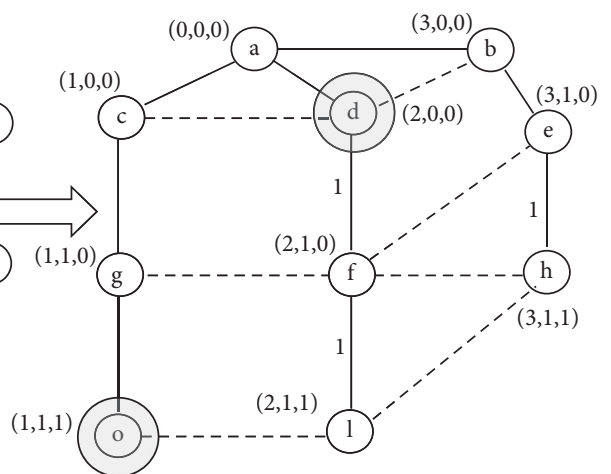

(c)

FIgURE 6: (a) Network topology. (b) Spanning tree and children numbering. (c) Coordinate set of each node given.

\section{(1) Begin;}

(2) while packet is not at the destination do

(3) for each neighbor of present node do

(4) find the first pair of different coordinates, comparing bit by bit, between neighbor and destination node; from there, count the number of nonzero coordinates from both CS to encounter distance;

(5) end

(6) compare found distances;

(7) forward packet to node with smallest distance to destination;

(8) end

Algorithm 1: Forwarding a packet to its destination. 


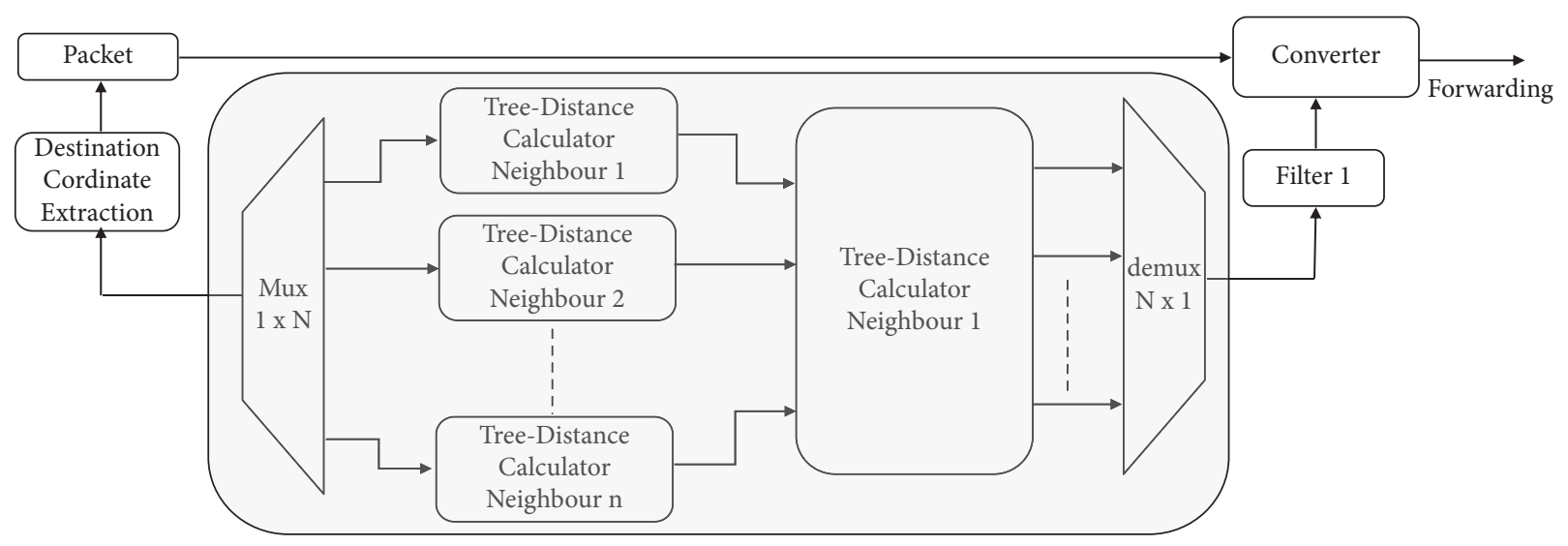

FIgURE 7: Geometric greedy router [25].

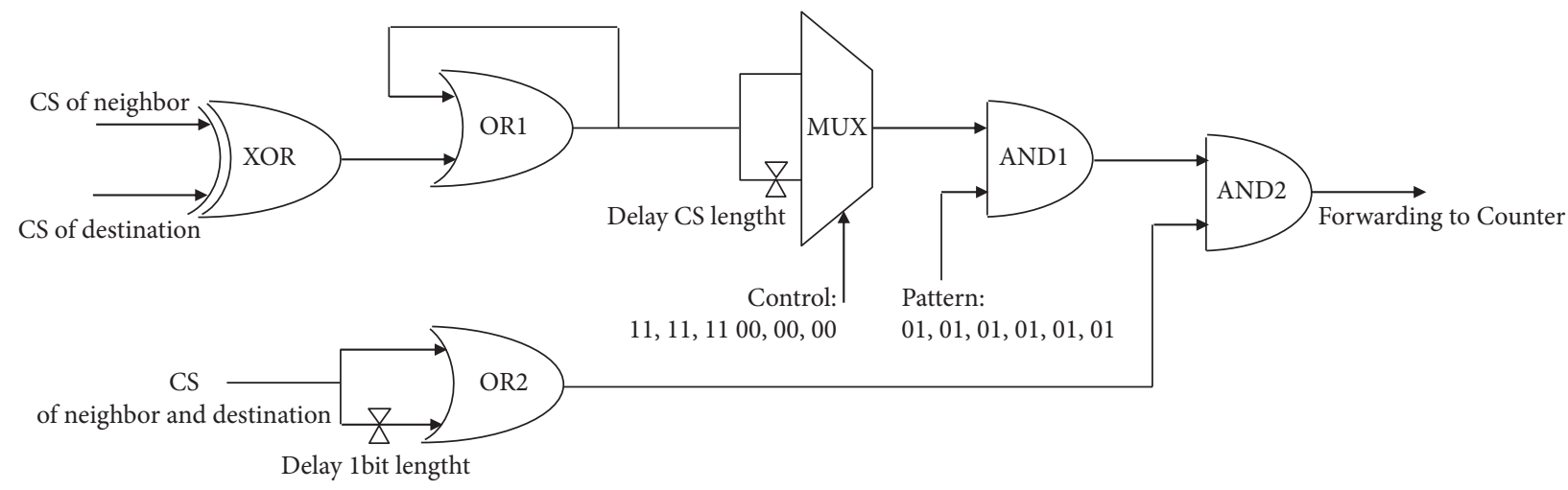

Figure 8: Circuit diagram of the distance calculator.

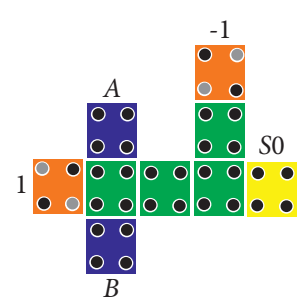

(a)

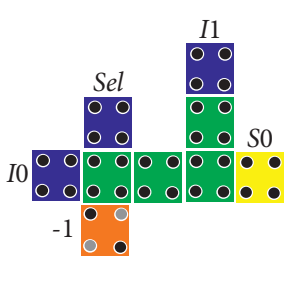

(b)

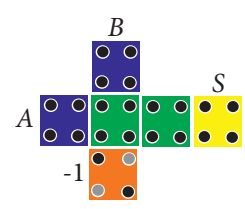

(c)

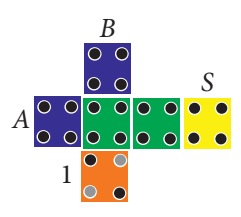

(d)

Figure 9: Basic logic gates of distance calculator module: (a) XOR; (b) 2:1 Mux; (c) And; (d) Or.

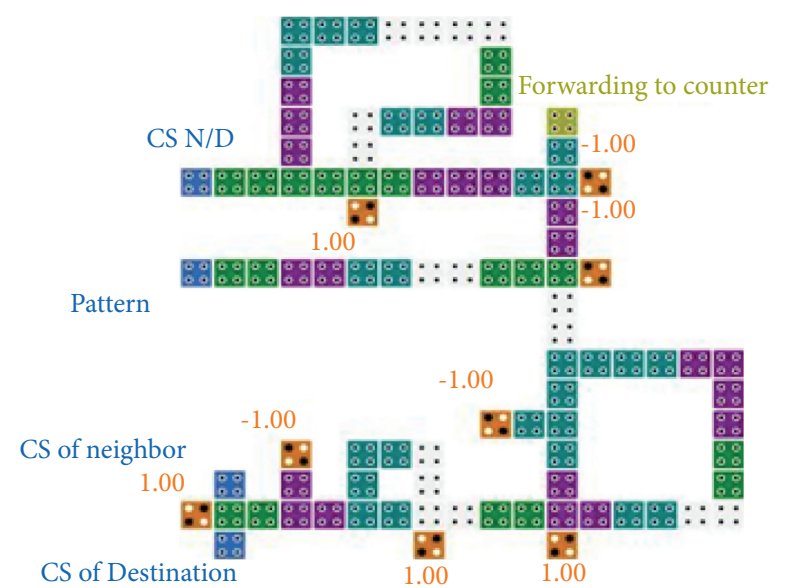

FIgURE 10: QCA architecture of the distance calculator module (DCM). 


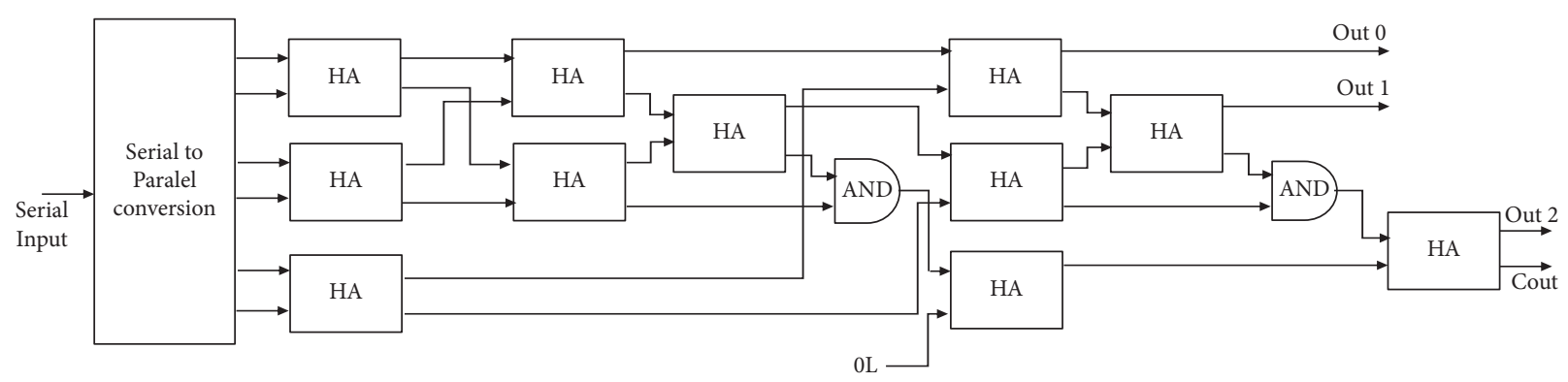

FIgURE 11: Architecture of the counter.
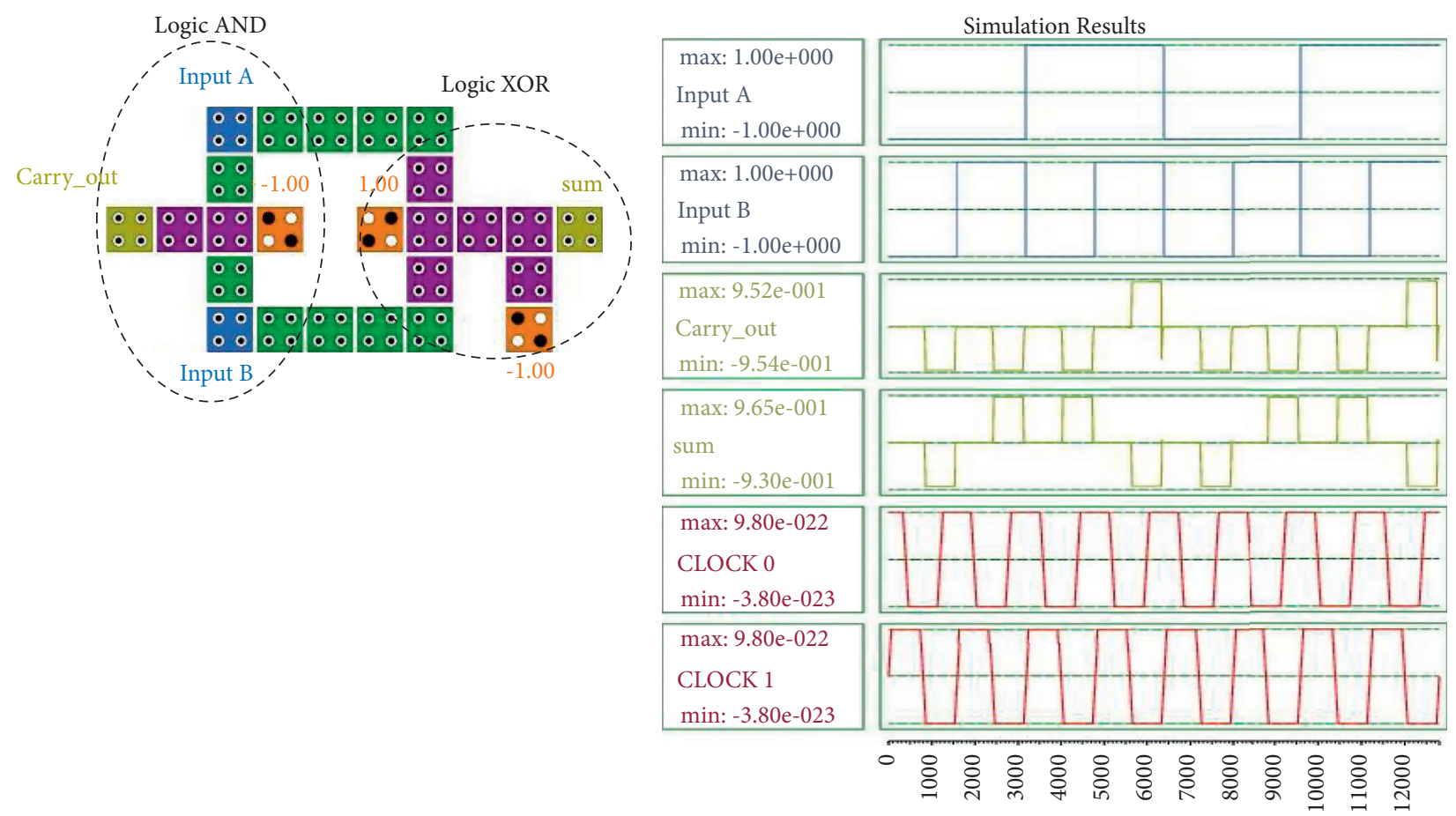

(a)

(b)

Figure 12: Half Adder. (a) Layout. (b) Simulation result.

A proposed realization of circuit diagram of distance calculator based on QCA technology is presented in Figure 10. The proposed architecture is composed by one 2 to 1 QCA multiplexer, two OR gates, two And gates, and one XOR gate.

The next step is to count the number of ' 1 ' in the output string that presents the number of marked coordinates. Figure 11 shows the architecture of the counter. The circuit, composed by a series-to-parallel converter, is based on eleven Half Adder circuits and two 'and' logic gates.

The next step is to count the number of ' 1 ' in the output string that presents the number of marked coordinates. Figure 11 shows the architecture of the counter. The circuit, composed by a series-to-parallel converter, is based on eleven Half Adder circuits and two 'and' logic gates.

Since the Half adder presents an essential component of the counter, in Figure 12(a) and 12(b), we present the optimized QCA layout and result simulation of this component.

An example of a tree with depth 3 is presented in Figure 13. We use a three coordinates to each CS. For tree nodes, we can attribute a maximum number of children of three, so two bits are enough to represent each coordinate.

The comparator circuit presents an essential submodule of the entire system. The comparator compares two binary 3 bits numbers $(A, B)$ and produces three outputs $(A>B$, $A=B$, and $A<B$ ). The logic circuit of the comparator is presented in Figure 14.

The proposed QCA layout of the comparator circuit is illustrated in Figure 15. The assembly of the submodules constitutes the greed router architecture (Figure 16). In the block diagram of the proposed architecture, we used two distance calculators and two counters to receive the coordinates of packet destination and neighbor. After the 


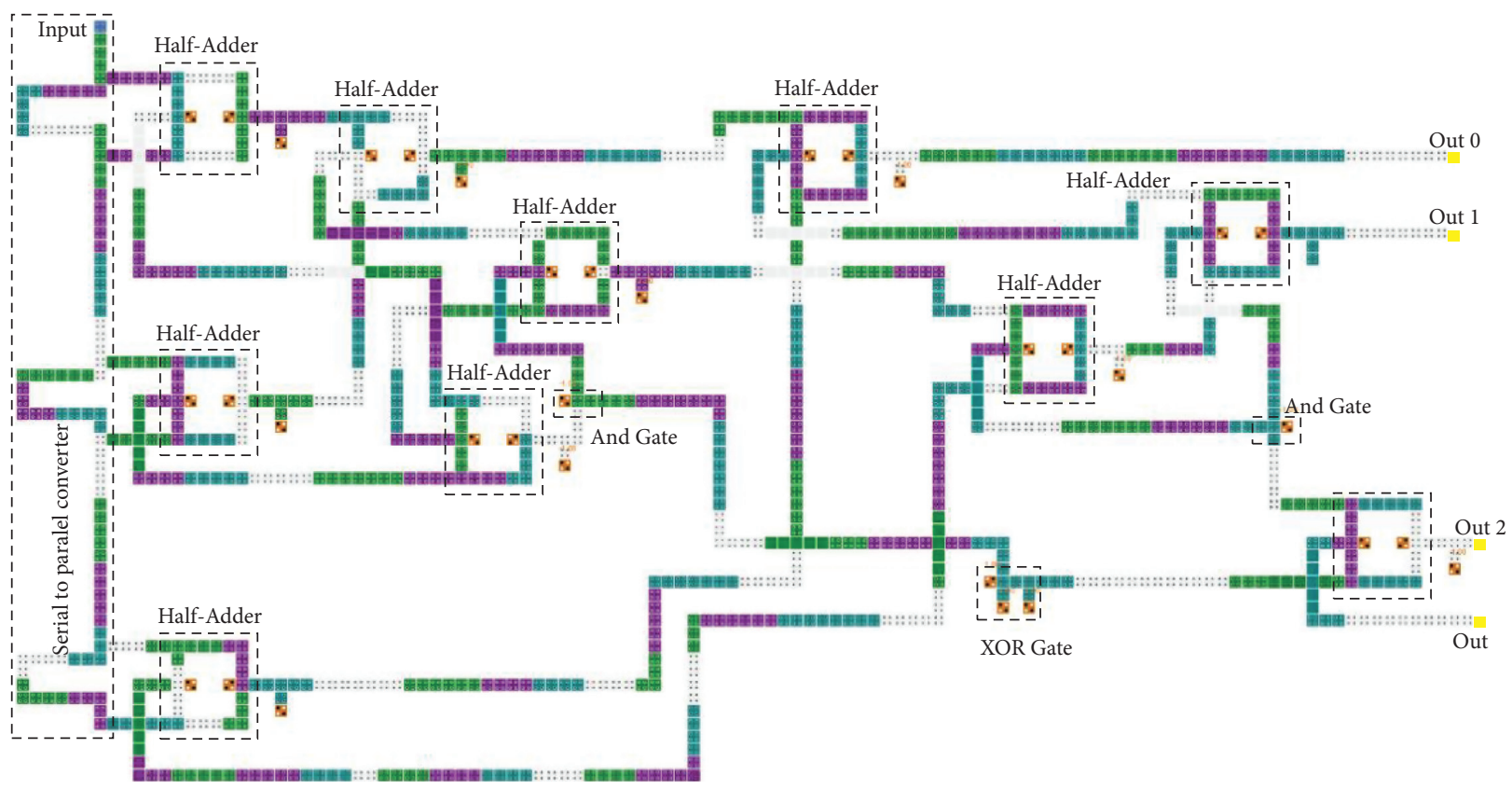

FIGURE 13: QCA architecture of the countermodule (CM).

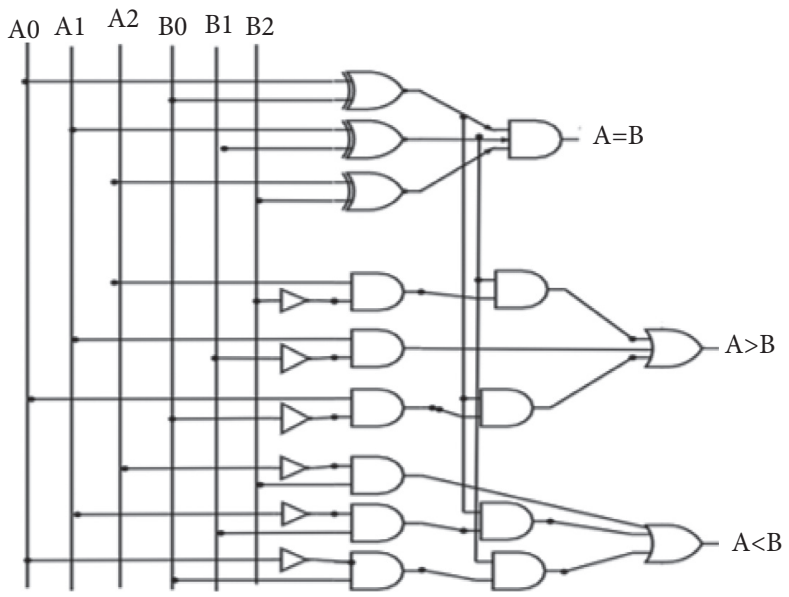

FIgURE 14: Comparator circuit.

counters, the results will be used by the comparator. The output will present the destination node for the packet.

The QCA architecture of the entire greed router is illustrated in Figure 17. The figure presents the inputs and outputs of the system.

\section{Result and Discussion}

The comparison between the proposed used logic gates and the existing architectures is given in Tables 1-3. In these tables, the complexity is given by the number of the required cells and the area is given by $\mu \mathrm{m}^{2}$.

The proposed QCA distance calculator layout is composed by 96 cells and occupied a surface of $0.313 \mu \mathrm{m}^{2}$. In [38], the authors have proposed a circuit composed by 154 cells and occupied a surface of $0.461 \mu \mathrm{m}^{2}$.
Half Adder presents the basic elements of the countermodule. For implementing the half adder in QCA technology, we have used one two-input XOR gate and one two-input AND gate.

Figure 12 shows the proposed layout of the QCA Half Adder circuit and the simulation result. Table 3 shows a comparison between the proposed Half Adder with the previous works. Half Adder circuit has lower computational complexity and better performances compared to existing ones.

The countercircuit is composed by ten Half Adder circuits and two AND logic gates. It occupies a surface of $1.73 \mu \mathrm{m}^{2}$. The proposed circuits are simulated using QCADesigner tool version 2.0.3 [40]. This allows users to do a custom layout and also verify QCA design by simulation. A nominal cell size of $18 \mathrm{~nm}$ by $18 \mathrm{~nm}$ is assumed. The 


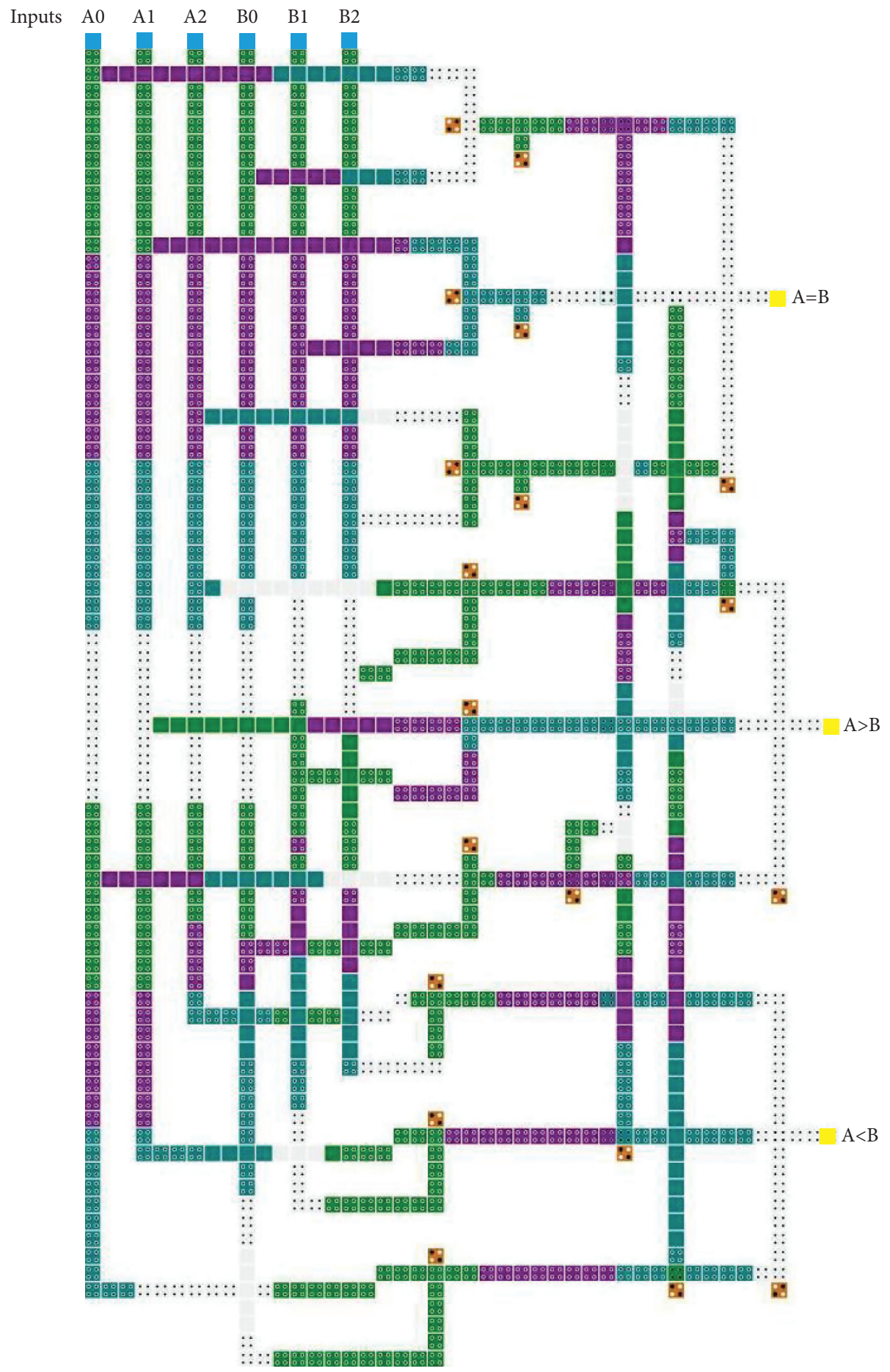

FIgURE 15: QCA architecture of the comparator (CMP). 


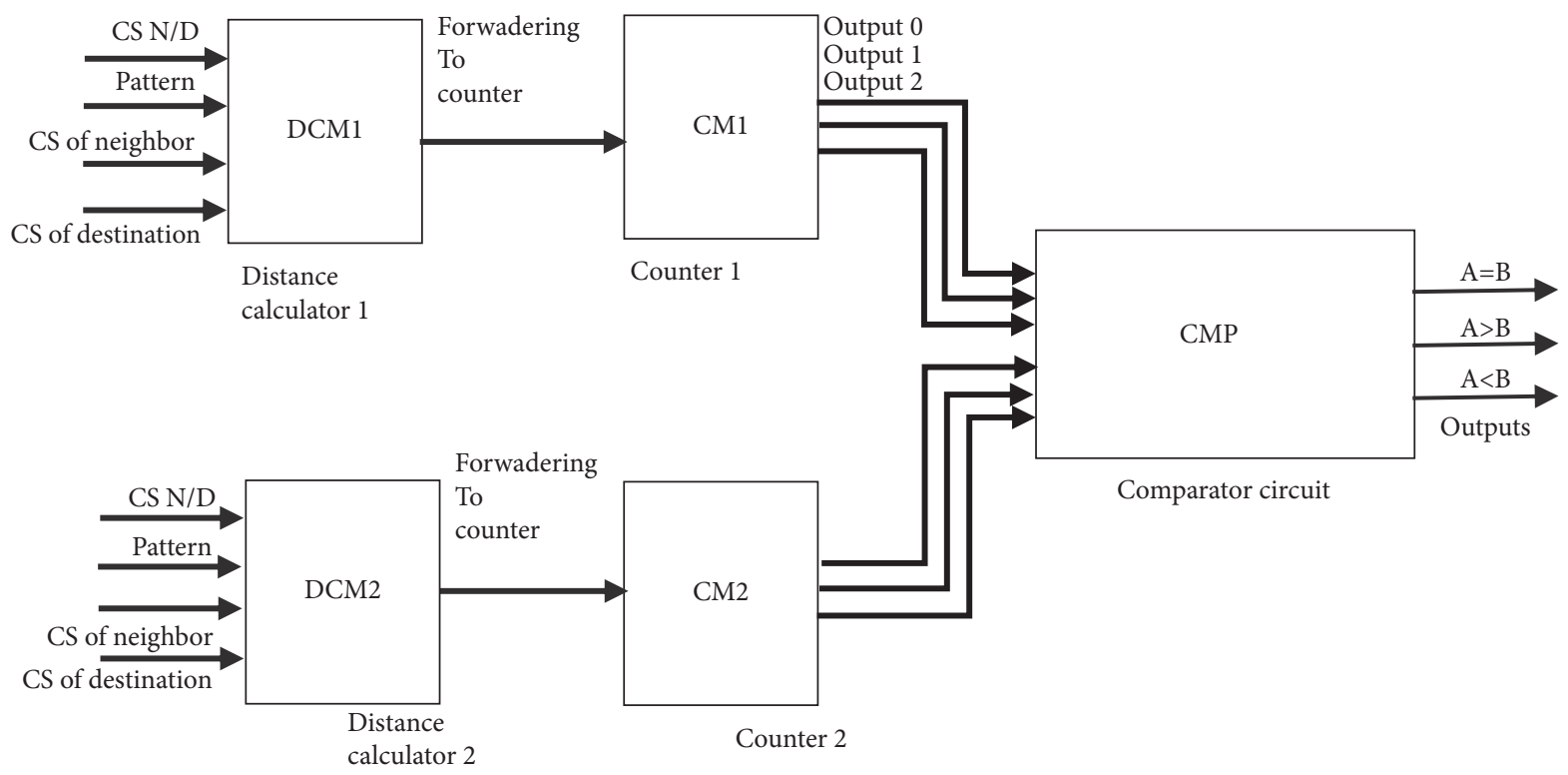

FigURE 16: Block diagram of the greed router.

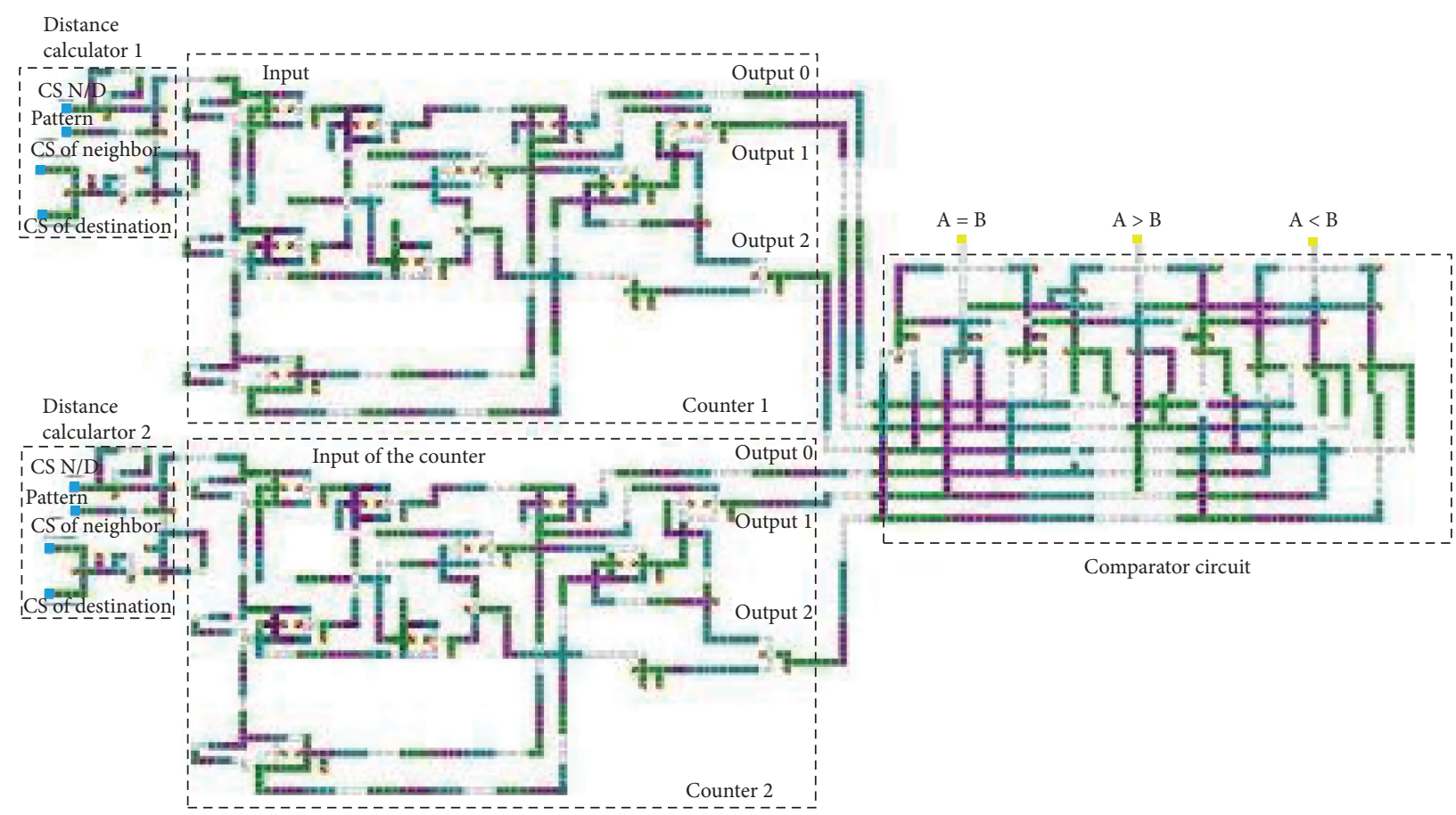

FIGURE 17: Block diagram of the greed router.

TABLE 1: Comparison results of the used 2-input digital "XOR" gate.

\begin{tabular}{lccc}
\hline XOR structure & Cell count & Surface $\left(\mu \mathrm{m}^{2}\right)$ & Crossover \\
\hline$[26]$ & 60 & 0.08 & Coplanar (rotated cells) \\
{$[27]$} & 29 & 0.03 & Not required \\
{$[28]$} & 28 & 0.02 & Coplanar \\
{$[29]$} & 12 & 0.0116 & Not required \\
{$[30]$} & 13 & 0.01 & Not required \\
{$[31]$} & 10 & 0.008 & Not required \\
Used & 9 & 0.008 & Not required \\
\hline
\end{tabular}


TABLE 2: Comparative result of $2: 1$ multiplexer.

\begin{tabular}{lccc}
\hline $2: 1$ mux & Cell count & Surface $\left(\mu \mathrm{m}^{2}\right)$ & Crossover \\
\hline$[32]$ & 26 & 0.02 & Coplanar \\
{$[33]$} & 29 & 0.02 & Coplanar \\
{$[34]$} & 28 & 0.02 & Coplanar \\
{$[35]$} & 12 & 0.02 & Coplanar \\
{$[36]$} & 13 & 0.01 & Coplanar \\
{$[37]$} & 12 & 0.02 & Without \\
Used & 9 & 0.0076 & Without \\
\hline
\end{tabular}

Table 3: Comparative result of Half Adder.

\begin{tabular}{lcc}
\hline Half Adder & Cell count & Surface $(\mu \mathrm{m} 2)$ \\
\hline$[39]$ & 77 & 0.08 \\
{$[40]$} & 62 & 0.08 \\
Proposed & 25 & 0.025 \\
\hline
\end{tabular}

TABLE 4: Simulation parameters.

\begin{tabular}{lc}
\hline Parameter & Value \\
\hline Cell size & $18 \mathrm{~nm}^{*} 18 \mathrm{~nm}$ \\
Dot & $5 \mathrm{~nm}$ \\
Cell separation & $2 \mathrm{~nm}$ \\
Simulation engine & Bistable approximation/coherence vector \\
Radius of effect & $65 \mathrm{~nm}$ \\
Number of samples & 12800 \\
Convergence tolerance & 0.001000 \\
Temperature & 1.000000 \\
Relative permittivity & 12.900000 \\
Clock high & $9.800000 e-022$ \\
Clock low & $3.800000 e-023$ \\
Clock shiftcty & 0.000000 \\
Separation & 2.000000 \\
Maximum iterations per sample & 11.500000 \\
Relaxation time & 100 \\
\hline
\end{tabular}

propagation delays from cell to cell required a maximum cell count in a clock zone.

The minimum separation between two different signal wires is width of two cells. We have used multilayer crossovers for wire crossings essentially depending on the complexity of the circuit. The essential QCA parameters are presented in Table 4.

\section{Conclusion}

In this paper, an optimized design of nanotechnology geometric greedy router architecture in the QCA technology was proposed. The GGR presents the principal components of the router, and this work presents an essential step in the building of QCA circuits for low power in digital nanocommunications systems. Our main focus is to propose a prototype of a very low-power GGR module optimized in terms of cell count and area. Results of simulation are shown by QCADesigner version 2.0.3. An interesting future work could be to develop several applications based on this GGR module.

\section{Data Availability}

The data used to support the findings of this study are available from the corresponding author upon request

\section{Conflicts of Interest}

The authors have no conflicts of interest.

\section{References}

[1] M. J. Shari_and M. Ahmadian, "Novel designs for digital gates based on single electron devices to overcome the traditional limitation on speed and bit error rate," Microelectronics Journal, vol. 73, pp. 12-17, 2018.

[2] A. Sadhu, K. Das, D. De, and M. R. Kanjilal, "Area-DelayEnergy aware SRAM memory cell and $\mathrm{M} \times \mathrm{N}$ parallel read/ write memory array design for quantum dot cellular automata," Microprocessors and Microsystems, vol. 72, p. 102944, 2020.

[3] F. Ahmad, S. Ahmed, V. Kakkar, G. M. Bhat, A. N. Bahar, and S. Wani, "Modular design of ultra-efficient reversible full adder-subtractor in QCA with power dissipation analysis," 
International Journal of Theoretical Physics, vol. 57, no. 9, pp. 2863-2880, 2018.

[4] T. Zhang, V. Pudi, and W. Liu, "New majority gate-based parallel BCD adder designs for quantum-dot cellular automata," IEEE Transactions on Circuits and Systems II: Express Briefs, vol. 66, no. 7, pp. 1232-1236, 2018.

[5] Z. Song, G. Xie, X. Cheng, L. Wang, and Y. Zhang, "An ultralow cost multilayer RAM in quantum-dot cellular automata," IEEE Transactions on Circuits and Systems II, 2020.

[6] A. N. Bahar and K. A. Wahid, "Design of an efficient $\mathrm{N} \times \mathrm{N}$ butterfly switching network in quantum-dot cellular automata (QCA)," IEEE Transactions on Nanotechnology, vol. 19, pp. 147-155, 2020.

[7] V. S. Kalogeiton, D. P. Papadopoulos, O. Liolis, V. A. Mardiris, G. C. Sirakoulis, and I. G. Karafyllidis, "Programmable crossbar quantum-dot cellular automata circuits," IEEE Transactions on Computer-Aided Design of Integrated Circuits and Systems, vol. 36, no. 8, pp. 1367-1380, 2016.

[8] S. M. Bhat and S. Ahmed, "Design of ultra-efficient reversible gate based 1-bit full adder in QCA with power dissipation analysis," International Journal of Theoretical Physics, vol. 58, no. 12, pp. 4042-4063, 2019.

[9] B. Bilal, S. Ahmed, and V. Kakkar, "Modular adder designs using optimal reversible and fault tolerant gates in fieldcoupled QCA nanocomputing," International Journal of Theoretical Physics, vol. 57, no. 5, pp. 1356-1375, 2018.

[10] M. G. Roshan and M. Gholami, "4-Bit serial shift register with reset ability and 4-bit LFSR in QCA technology using minimum number of cells and delay," Computers \& Electrical Engineering, vol. 78, pp. 449-462, 2019.

[11] T. Li and R. Kornovich, "An optimized design of serial-inputserial-output (SISO) and parallel-input-parallel-output (PIPO) shift registers based on quantum dot cellular automata nanotechnology," International Journal of Theoretical Physics, vol. 58, no. 11, pp. 3684-3693, 2019.

[12] S. Sahhaf, W. Tavernier, D. Colle, M. Pickavet, and P. Demeester, "Experimental validation of resilient tree-based greedy geometric routing," Computer Networks, vol. 82, pp. 156-171, 2015.

[13] L. Touil, I. Gassoumi, R. Lajimi, and B. Ouni, "An efficient design of BinDCT in quantum-dot cellular automata (QCA) technology IET image processing"” ", vol. 12, no. 6, pp. 1020-1030, 2018.

[14] I. Gassoumi, L. Touil, and B. Ouni, "Design of efficient quantum Dot cellular automata (QCA) multiply accumulate (MAC) unit with power dissipation analysis," IET Circuits, Devices and Systems, vol. 13, no. 4, pp. 534-543, 2019.

[15] W. H. Liao, J. P. Sheu, and Y. C. T. Grid, "A fully locationaware routing protocol for mobile ad hoc networks," Telecommunication Systems, vol. 18, no. 1-3, pp. 37-60, 2001.

[16] S. Sahhaf, A. Dixit, W. Tavernier, D. Colle, M. Pickavet, and P. Demeester, "All-optical tree-based greedy router using optical logic gates and optical flip-flops," Photonic Network Communications, vol. 35, no. 1, pp. 109-121, 2018.

[17] R. Sarkar, X. Yin, J. Gao, F. Luo, and X. D. Gu, "Greedy routing with guaranteed delivery using ricci ows," in Proceedings of the International Conference on Information Processing in Sensor Networks, pp. 121-132, New York, NY, USA, November 2009.

[18] A. Maghsoudlou, M. St-Hilaire, and T. Kunz, "A survey on geographic routing protocols for mobile ad hoc networks, systems and computer engineering," Technical Report SCE11-03, Carleton University, Ottawa, Canada, 2011.
[19] S. Sahhaf, W. Tavernier, D. Colle, M. Pickavet, and P. Demeester, "Experimental validation of resilient tree-based greedy geometric routing," Computer Networks, vol. 82, pp. 156-171, 2015.

[20] R. Flury, S. V. Pemmaraju, and R. Wattenhofer, "Greedy routing with bounded stretch," in Proceedings of the IEEE INFOCOM, pp. 1737-1745, IEEE, Rio de Janeiro, Brazil, April 2009.

[21] C. Westphal and G. Pei, "Scalable routing via greedy embedding," in Proceedings of the IEEE INFOCOM, pp. 28262830, IEEE, Rio de Janeiro, Brazil, April 2009.

[22] M. Tang, H. Chen, G. Zhang, and J. Yang, "Tree cover based geographic routing with guaranteed delivery," in Proceedings of the 2010 IEEE International Conference on Communications, pp. 1-5, IEEE, Cape Town, South Africa, November 2010.

[23] J. Herzen, C. Westphal, and P. Thiran, "Scalable routing easy as pie: a practical isometric embedding protocol," in Proceedings of the 2011 19th IEEE International Conference on Network Protocols, pp. 49-58, Vancouver, BC, USA, October 2011.

[24] S. Sahhaf, W. Tavernier, D. Colle, M. Pickavet, and P. Demeester, "Single failure resiliency in greedy routing," in Proceedings of the 2013 9th International Conference on the Design of Reliable Communication Networks (DRCN), pp. 565-313, 2013.

[25] L. G. L. Vieira, L. F. M. Vieira, M. Augusto, M. Vieira, and V. Paranaiba., "Neto "geometric greedy router in quantumdot cellular automata", computer science," Aeu-international Journal of Electronics and Communications, vol. 18, Article ID 153498, 2021.

[26] S. Hashemi, R. Farazkish, and K. Navi, "New quantum dot cellular automata cell arrangements," Journal of Computational and Theoretical Nanoscience, vol. 10, no. 4, pp. 798-809, 2013.

[27] A. Chabi, S. Sayedsalehi, S. Angizi, and K. Navi, "Efficient QCA exclusive-or and multiplexer circuits based on a NanoelectronicCompatible designing approach," Hindawi Publishing Corporation International Scholarly Research Notices, vol. 2014, p. 9, Article ID 463967, 2014.

[28] G. Singh, R. K. Sarin, and B. Raj, “A novel robust exclusive-OR function implementation in QCA nanotechnology with energy dissipation analysis," Journal of Computational Electronics, vol. 15, no. 2, pp. 455-465, 2016.

[29] A. N. Bahar, S. Waheed, N. Hossain, and Md. Asaduzzaman, "A novel 3- input XOR function implementation in quantum dot-cellular automata with energy dissipation analysis," Alexandria Engineering Journal, vol. 57, pp. 729-738, 2018.

[30] A. M. Chabi, A. Roohi, H. Khademolhosseini et al., "Towards ultra-efficient QCA reversible circuits," Microprocessors and Microsystems, vol. 49, pp. 127-138, 2017.

[31] R. Laajimi, A. Ajimi, L. Touil, and A. N. Bahar, "A novel design for XOR gate used for quantum-dot cellular automata (QCA) to create a revolution in nanotechnology structure"," International Journal of Advanced Computer Science and Applications, vol. 8, no. 10, 2017.

[32] R. S. N. Nadoochen and M. Kianpour, "A novel QCA implementation of MUX-based universal shift register," Journal of Computational Electronics, vol. 13, no. 1, pp. 198210, 2014.

[33] B. Sen, M. Goswami, S. Mazumdar, and B. K. Sikdar, "Towards modular design of reliable quantum-dot cellular automata logic circuit using multiplexers," Computers \& Electrical Engineering, vol. 45, pp. 42-54, 2016. 
[34] B. Sen, M. Dutta, M. Goswami, and B. K. Sikdar, "Modular Design of testable reversible ALU by QCA multiplexer with increase in programmability," Microelectronics Journal, vol. 45, no. 11, pp. 1522-1532, 2014.

[35] B. Sen, M. Dutta, D. Saran, and B. K. Sikdar, "An efficient multiplexer in quantum-dot cellular automata," Progress in VLSI Design and Test, vol. 7373, pp. 350-351, 2012.

[36] H. Rashidi, A. Rezai, and S. Soltany, "High-performance multiplexer architecture for quantum-dot cellular automata," Journal of Computational Electronics, vol. 15, no. 3, pp. 968-981, 2016.

[37] M. Naji Asfestani and S. Rasouli Heikalabad, "A unique structure for the multiplexer in quantum-dot cellular automata to create a revolution in design of nanostructures," Physica B: Condensed Matter, vol. 512, pp. 91-99, 2017.

[38] L. G. L. Vieira, L. F. M. Vieira, M. A. M. Vieira, and O. P. V. Neto, "Geometric greedy router in quantum-dot cellular automata," AEU - International Journal of Electronics and Communications, vol. 128, p. 153498, 2021.

[39] S. Craig, P. Lent, and T. Douglas, "A device architecture for computing with quantum dots," Proceedings of the IEEE, vol. 85, no. 4, pp. 541-557, 1997.

[40] K. Walus, T. J. Dysart, G. A. Jullien, and R. A. Budiman, "QCADesigner: a rapid design and simulation tool for quantum-dot cellular automata," IEEE Transactions on Nanotechnology, vol. 3, no. 1, pp. 26-31, 2004. 